\title{
A new differentially weighted operator splitting Monte Carlo method for aerosol dynamics
}

\author{
H. Liu \& T. L. Chan \\ Department of Mechanical Engineering, \\ The Hong Kong Polytechnic University, Hong Kong
}

\begin{abstract}
Monte Carlo method is a stochastic scheme which replicates the formation, transport and dynamic behaviour of simulation particles with stochastic method. It is based on probabilities rather than solving directly the general dynamic equation. Differentially weighted Monte Carlo method is quite efficient and more practical than using the concept of each simulation particle being equally weighted with a number of real particles. In the present study, a new differentially weighted operator splitting Monte Carlo (DWOSMC) method is developed. In order to increase the computational efficiency of simulating the aerosol processes (i.e., nucleation, coagulation and condensation), an operator splitting technique is used to combine stochastic and deterministic methods. This new DWOSMC method is fully validated through four typical cases considering different aerosol dynamic processes. The results obtained from DWOSMC method agree well with the analytical solutions, which proves very high computational efficiency and accuracy of using DWOSMC method in solving simultaneous aerosol dynamic processes.

Keywords: differentially weighted Monte Carlo, operator splitting, aerosol dynamics.
\end{abstract}

\section{Introduction}

The dynamic behaviours of aerosol particles are of great importance in our life, as well as in many scientific and engineering fields including atmospheric sciences, air pollution and control, industrial production, combustion and chemical sciences, etc. [1]. Atmospheric aerosols are associated with plenty of air pollution problems. They bring many negative effects to our lives including adverse health 
effects, visibility reduction and global climate change [2]. The sources of fine particle pollution are related to human activities, like the automotive and industrial emissions [3]. In order to further understand the aerosol processes, more attention has been drawn to the study of aerosol dynamics in recent years. Different kinds of numerical models have been developed to simulate aerosol dynamic processes including coagulation, nucleation, condensation, etc. [4, 5].

Monte Carlo method is a stochastic scheme which replicates the formation, transport and dynamic behaviour of simulation particles with stochastic method based on probabilities rather than solves directly the general dynamic equation. Zhao et al. [6] proposed a differentially weighted Monte Carlo method, which is quite efficient and more practical than using the concept of each simulation particle being equally weighted with a number of real particles. In order to increase the efficiency of simulating all the aerosol processes (i.e., coagulation, nucleation and condensation), Zhou et al. [7] used the operator splitting technique to combine stochastic and deterministic methods. Liu and Chan [8] have recently developed a stochastically weighted operator splitting Monte Carlo method and have demonstrated its computational capability and efficiency. In the present study, a new differentially weighted operator splitting Monte Carlo (DWOSMC) method based on the idea of operator splitting and a different weight for each simulation particle is presented. The purpose of this new method aims to solve aerosol dynamic processes with high computational accuracy and efficiency.

\section{Methodology}

\subsection{General Dynamics Equation}

The dynamic behaviour of an aerosol can be expressed in the general dynamic equation (GDE) [1,7],

$$
\frac{\partial n}{\partial t}+\nabla \cdot n \vec{u}=\nabla \cdot D \nabla n+\left[\frac{\partial n}{\partial t}\right]_{\mathrm{nuc} 1}+\left[\frac{\partial n}{\partial t}\right]_{\mathrm{coag}}+\left[\frac{\partial n}{\partial t}\right]_{\mathrm{cond}}
$$

where $n$ is the particle size distribution function, $\vec{u}$ is the flow velocity of gas, $D$ is the diffusion coefficient.

Ignoring effects of convection and diffusion of the aerosols, the GDE becomes,

$$
\frac{\partial n}{\partial t}=\frac{1}{2} \int_{0}^{v} \beta(v, \tilde{v}) n(\tilde{v}) n(v-\tilde{v}) \mathrm{d} \tilde{v}-\int_{0}^{\infty} \beta(v, \tilde{v}) n(\tilde{v}) n(\tilde{v}) d \tilde{v}+\frac{\partial\left(I_{0} n\right)}{\partial v}(v, t)+\delta\left(v_{0}, v\right) J_{0}(t)
$$

where $\beta(v, \tilde{v}), I_{0}(v, t)$ and $J_{0}(t)$ are the coagulation, condensation and nucleation kernels, respectively [9].

\subsection{Algorithm formulation}

The concept of weighting simulation particles [10] has been widely used by Monte Carlo method to meet the challenge between the large number of real particles and the restriction of computational ability. The developed differentially weighted time driven Monte Carlo (DWMC) method by Zhao et al. [6] is used herein. 
Specifically, each simulation particle is differentially weighted with $w_{i}(i=0,1$, $2 \ldots n$ ) real particles, $n$ is the number of simulation particles. For the simulation of coagulation process, when simulation particles $i$ and $j$ coagulate with each other, the previous ones are replaced by two new simulation particles. They are formulated [11] as follows:

$$
\begin{aligned}
& \text { if } w_{i}=w_{j},\left\{\begin{array}{c}
w_{i}^{\prime}=w_{i} / 2 ; v_{i}^{\prime}=v_{i}+v_{j} ; \\
w_{j}^{\prime}=w_{j} / 2 ; v_{j}^{\prime}=v_{i}+v_{j} ;
\end{array}\right. \\
& \text { if } w_{i} \neq w_{j},\left\{\begin{array}{c}
w_{i}^{\prime}=\max \left(w_{i}, w_{j}\right)-\min \left(w_{i}, w_{j}\right) ; v_{i}^{\prime}=\left.v_{m}\right|_{w_{m}=\max \left(w_{i}, w_{j}\right)} \\
w_{\mathrm{j}}^{\prime}=\min \left(w_{i}, w_{j}\right) ; v_{j}^{\prime}=v_{i}+v_{j} ;
\end{array}\right.
\end{aligned}
$$

where $w_{i}^{\prime}, w_{j}^{\prime}, v_{i}^{\prime}$ and $v_{j}^{\prime}$ are the new values of the weight or the volume of simulation particle $i$ and $j$ after the coagulation event.

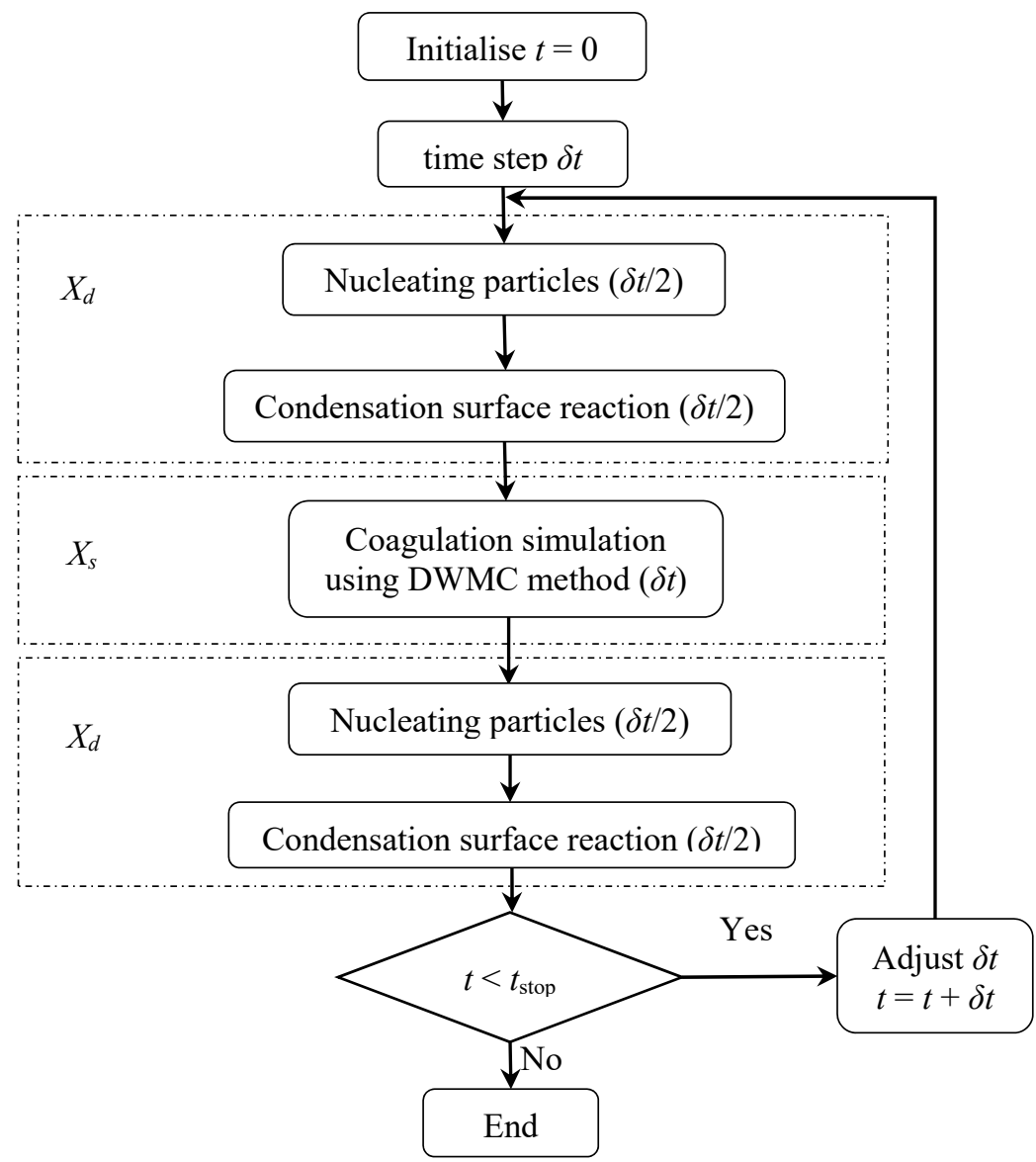

Figure 1: Flowchart of DWOSMC. 
Fig. 1 shows the flowchart of DWOSMC including all of the aerosol dynamic processes (i.e., coagulation, nucleation, and condensation). The second order operator splitting in eqn. (3) is presented.

The differentially weighted operator splitting Monte Carlo (DWOSMC) method is developed here, for the operator splitting method makes it very efficient and flexible to combine stochastic and deterministic methods. In the present study, the stochastic Monte Carlo method is utilised for the coagulation process, while the deterministic method is used for nucleation and surface growth processes. The second order Strang scheme [12] is applied herein, which can be expressed in the following equation:

$$
\exp (\delta t X)=\exp \left(\frac{1}{2} \delta t X_{\mathrm{d}}\right) \exp \left(\delta t X_{\mathrm{s}}\right) \exp \left(\frac{1}{2} \delta t X_{\mathrm{d}}\right)+\mathcal{O}\left(\delta t^{3}\right)
$$

where $X$ denotes the total aerosol dynamic process, $X_{\mathrm{d}}$ denotes nucleation and surface growth processes, which is solved by deterministic integration method, and $X_{\mathrm{s}}$ denotes coagulation process, which is modelled by the stochastic method, $\delta t$ denotes one-time step [7].

\subsubsection{Test problem for Cases I and II: simultaneous coagulation and condensation processes}

The DWOSMC method is firstly validated by considering simultaneous coagulation and condensation processes by the corresponding analytical solutions [13]. Two test cases [14] are considered for both coagulation and condensation processes occurring simultaneously. The simultaneous coagulation and condensation parameters, initial conditions and analytical solutions for Cases I and II are given in table 1 .

Table 1: Simultaneous coagulation and condensation parameters, initial conditions and analytical solutions used for Cases I and II.

\begin{tabular}{|c|c|c|c|}
\hline Test cases & Parameters & Analytical solution & Initial conditions \\
\hline \multirow{2}{*}{ I } & $K=K_{0}$ & $N(t)=\frac{N_{0}}{1+K_{0} N_{0} t / 2}$ & $K_{0}=5 \times 10^{-7}\left(\mathrm{~m}^{3} \mathrm{~s}^{-1}\right)$ \\
& $I=\sigma_{0}$ & $\varnothing(t)=\emptyset_{0}\left[1+\frac{2 \sigma_{0}}{K_{0} \emptyset_{0}} \ln \left(\frac{N_{0}}{N}\right)\right]$ & $\sigma_{0}=1 \times 10^{-5}\left(\mathrm{~s}^{-1}\right)$ \\
\hline II & $\begin{array}{c}K_{1}(u+v) \\
I=\sigma_{1} v\end{array}$ & $N(t)=N_{0} \exp \left[-\frac{K_{1} \emptyset_{0}}{\sigma_{1}}\left(\exp \left(\sigma_{1} t\right)-1\right)\right]$ & $\begin{array}{c}K_{1}=1 \times 10^{17}\left(\mathrm{~s}^{-1}\right) \\
\sigma_{1}=0.5\left(\mathrm{~s}^{-1}\right)\end{array}$ \\
\hline
\end{tabular}

The coagulation and condensation kernels are respectively represented by $K$ and $I . N(t)$ and $N_{0}$ represent the particle number concentration and initial particle number concentration, respectively. $\varnothing(t)$ and $\varnothing_{0}$ represent the total volume concentration and initial total volume concentration of the particles, respectively [14]. The constant coagulation and condensation kernels are considered for Case I. The coagulation kernel which is highly related to the volumes of the particles, $u$ 
and $v$, and the condensation kernel which is highly related to the volume of the particle, $v$, are considered for Case II.

\subsubsection{Test problem for Case III: simultaneous coagulation and nucleation processes}

This problem considers simultaneous nucleation and coagulation processes. The constant nucleation and coagulation kernels are considered for Case III and all newly nucleated particles would have the same diameter. The parameters, initial conditions and analytical solutions [15] are given in table 2. The coagulation and nucleation kernels are respectively represented by $K$ and $J . N$ and $V$ represent the number concentration and total volume concentration of particles, respectively.

Table 2: Parameters of initial conditions and analytical solutions used for Cases III and IV, where $\tau=t \sqrt{2 A J_{0}}, B=1 / N_{0} \sqrt{2 J_{0} / A}$, $E=D C_{0} / A N_{0}$.

\begin{tabular}{|c|c|c|c|}
\hline Test cases & Parameters & Analytical solution & Initial conditions \\
\hline III & $\begin{array}{l}K=A \\
J=J_{0}\end{array}$ & $\begin{array}{c}\frac{N}{N_{0}}=B \frac{1+B \tan (\tau / 2)}{\tanh (\tau / 2)+B} \\
\frac{V}{V_{0}}=1+\frac{1}{2} B \tau\end{array}$ & $\begin{array}{l}A=4 \times 10^{-28}\left(\mathrm{~m}^{3} \mathrm{~s}^{-1}\right) \\
J_{0}=1.91 \times 10^{28}\left(\mathrm{~s}^{-1}\right)\end{array}$ \\
\hline IV & $\begin{array}{c}K=A \\
J=J_{0} \\
I=D\end{array}$ & $\begin{array}{c}\frac{N}{N_{0}}=B \frac{1+B \tan (\tau / 2)}{\tanh (\tau / 2)+B} \\
\frac{V}{V_{0}}=1+\left(\frac{1}{2} B+E\right) \tau+ \\
2 E \ln \left(\frac{1+\exp (-\tau)}{2}+\frac{1-\exp (-\tau)}{2 B}\right)\end{array}$ & $\begin{array}{l}A=4 \times 10^{-28}\left(\mathrm{~m}^{3} \mathrm{~s}^{-1}\right) \\
J_{0}=1.91 \times 10^{28}\left(\mathrm{~s}^{-1}\right) \\
D=2 \times 10^{-28}\left(\mathrm{~m}^{3} \mathrm{~s}^{-1}\right) \\
C_{0}=1.91 \times 10^{28}\left(\mathrm{~s}^{-1}\right)\end{array}$ \\
\hline
\end{tabular}

\subsubsection{Test problem for Case IV: simultaneous coagulation, nucleation and condensation processes}

This problem includes all the aerosol dynamic processes (i.e., nucleation, coagulation and condensation). The constant nucleation, coagulation and condensation kernels are considered for Case IV. The simultaneous nucleation, coagulation and condensation parameters, initial conditions and analytical solutions [15] for Case IV are given in table 2.

\subsection{Evaluation of simulation results}

The relative error, $\varepsilon$ is used to evaluate the accuracy of the simulation results, and is defined as follows:

$$
\varepsilon=\left|A(t)-A_{0}(t)\right| / A_{0}(t)
$$

where $A(t)$ and $A_{0}(t)$ are the simulation value and the analytical value at time $t$, respectively. 


\section{Results and discussion}

\subsection{Test problem for Cases I and II: simultaneous coagulation and condensation processes}

For the test problem in Cases I and II, 100,000 particles and the order of $10^{-17} \mathrm{~m}^{3}$ are used as the initial particle population, $N_{0}$ and the initial volume of particles, $V_{0}$ respectively. Case I is observed for $200 \mathrm{~s}$, and Case II is observed for $3.5 \mathrm{~s}$. An increasing number of simulation particles (i.e., $N_{\mathrm{p}}=500,800$ and 1000) is used for the calculation.

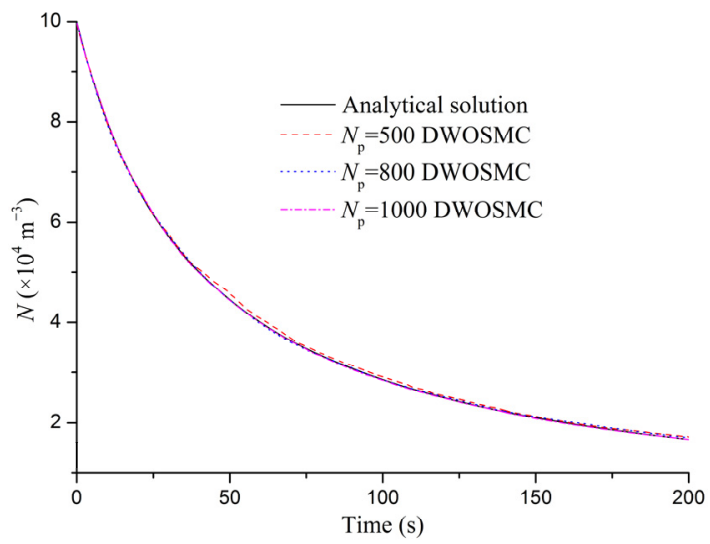

(a) Case I

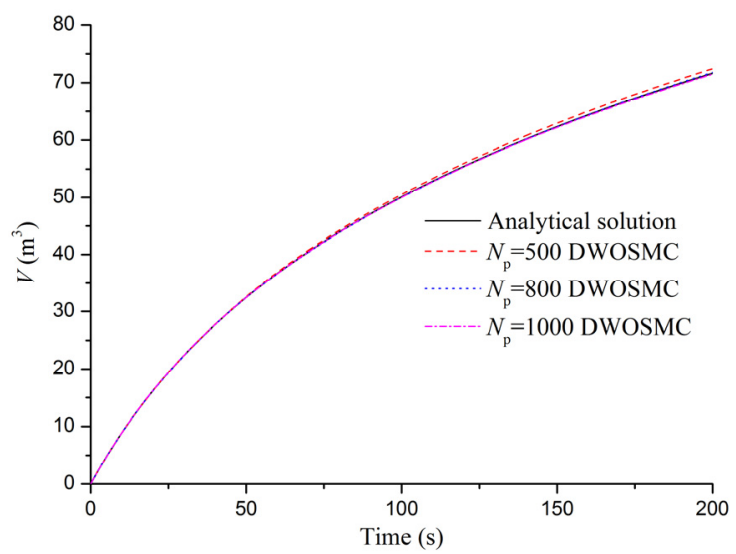

(b) Case I

Figure 2: Variations of particle number concentration, $\mathrm{N}$ and total particle volume concentration, $V$ as the function of time for different simultaneous coagulation and condensation processes in Cases I and II using DWOSMC method and analytical solutions [13]. 


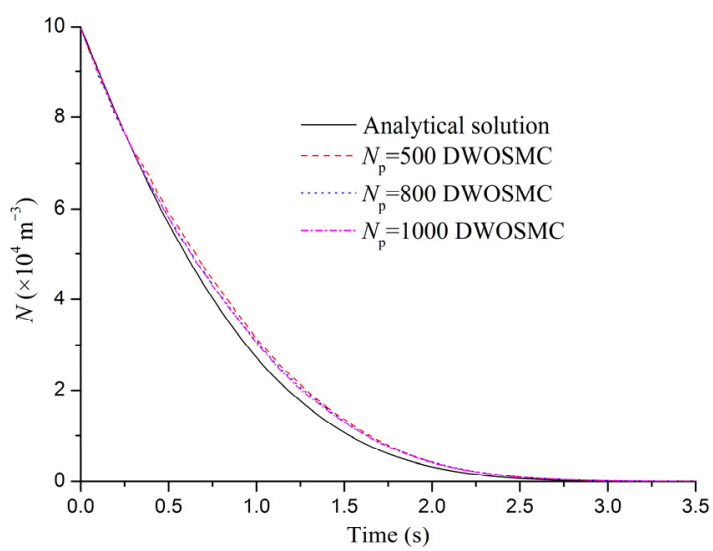

(c) Case II

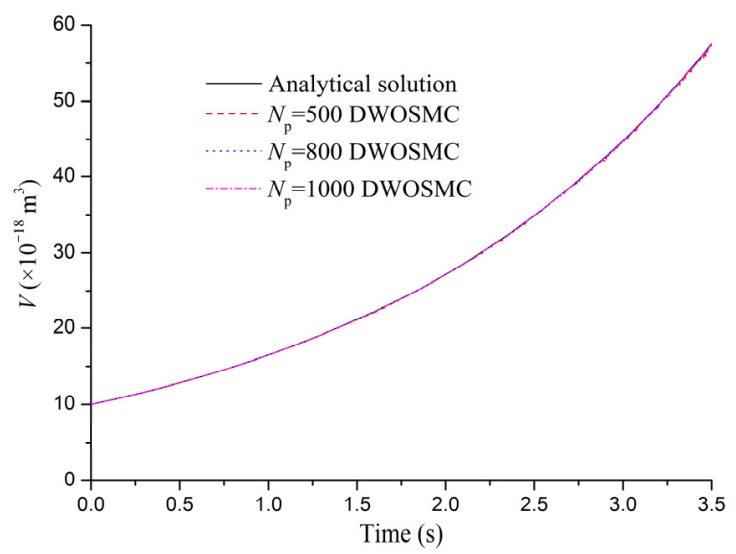

(d) Case II

Figure 2: Continued.

Generally, the particle number concentration decreases over time but the total particle volume concentration increases over time for simultaneous coagulation and condensation processes in Cases I and II (as shown in fig. 2). From table 1, the particle number concentration depends only on coagulation kernel for Case I but not the condensation kernel. While for Case II, both the coagulation and condensation kernels affect the particle number concentration. For Case II, the total particle volume concentration depends only on condensation kernel but not the total particle volume. While for Case I, both the coagulation and condensation kernels affect the total particle volume concentration.

Fig. 2 shows that the results obtained from DWOSMC method agree well with the analytical solutions for Case I when only 800 simulation particles are used. But there are slight differences between the simulation results and the analytical solution for the particle number concentration in Case II. It is because the particle 
number changes quite significantly over time. While the total particle volume concentration obtained from DWOSMC method agrees well with the analytical solution for Case II.

\subsection{Test problem for Case III: simultaneous coagulation and nucleation processes}

For the test problem in Case III, $1.91 \times 10^{23}$ particles $/ \mathrm{m}^{3}$ is used as the initial particle population and $10^{-27} \mathrm{~m}^{3}$ is used as the initial volume of each particle. It is observed up to $45 \mathrm{~ms}$. The variations of ratios of simulation results to initial values for the particle number concentration, $N / N_{0}$ and particle volume concentration, $V / V_{0}$ are shown in fig. 3. They show approximately linear relationship within an extreme

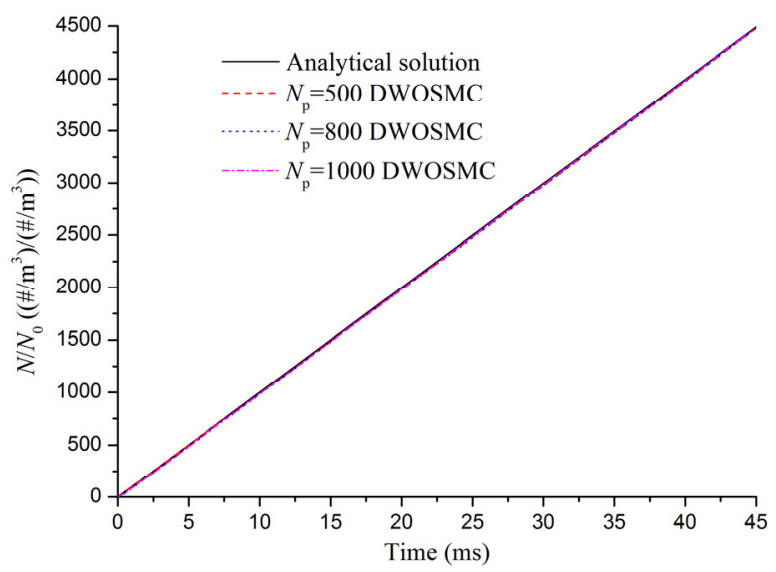

(a)

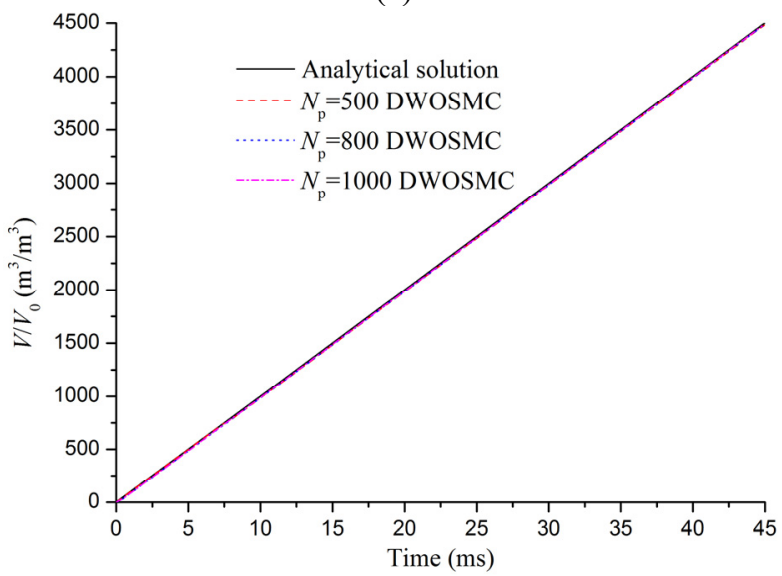

(b)

Figure 3: Variations of (a) $N / N_{0}$ and (b) $V / V_{0}$ as the functions of time for simultaneous coagulation and nucleation processes in Case III using DWOSMC method and analytical solution [15]. 
short period of time, $45 \mathrm{~ms}$. For simultaneous nucleation and coagulation processes, the results obtained from DWOSMC method agree well with the analytical solutions. Even with only 500 simulation particles, the simulation results show very small fluctuations and errors.

\subsection{Test problem for Case IV: simultaneous coagulation, nucleation and condensation processes}

For the test problem in Case IV, $1.91 \times 10^{23}$ particles $/ \mathrm{m}^{3}$ is used as the initial particle population and $10^{-27} \mathrm{~m}^{3}$ is used as the initial volume of each particle. The variations of ratios of simulation results to initial values for the particle number concentration, $N / N_{0}$ and particle volume concentration, $V / V_{0}$ [15] within an extreme short period of time, $45 \mathrm{~ms}$ are shown in fig. 4 . They show approximately

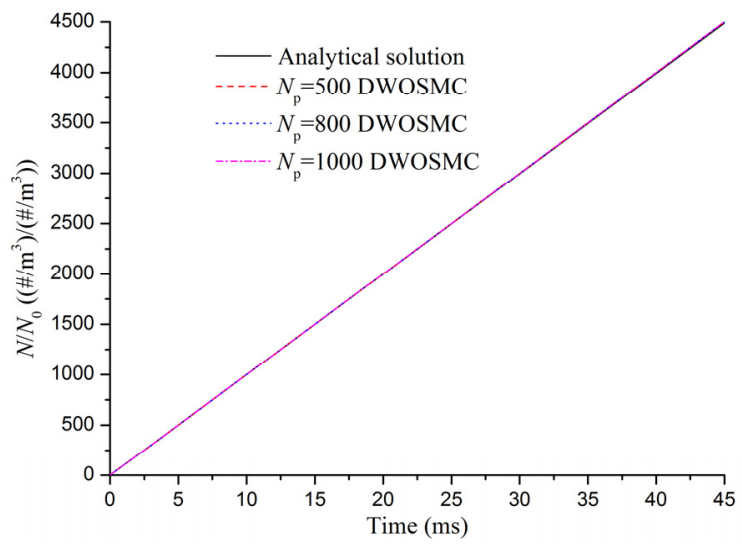

(a)

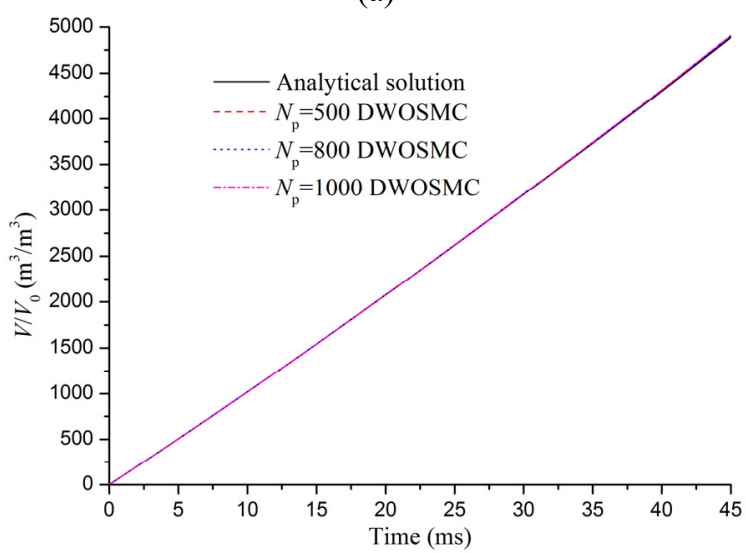

(b)

Figure 4: Variations of (a) $N / N_{0}$ and (b) $V / V_{0}$ as the functions of time for simultaneous nucleation, coagulation and condensation processes coagulation and nucleation processes in Case IV using DWOSMC method and analytical solution [15]. 
linear relationship within an extreme short period of time, $45 \mathrm{~ms}$. Even with only 500 simulation particles, the simulation results show excellent agreement with the analytical solutions for simultaneous nucleation, coagulation and condensation processes.

\subsection{Computational efficiency and accuracy analysis}

The maximum relative error and the time consumption using DWOSMC method for different cases are shown in table 3 .

Table 3: Computational efficiency and accuracy analysis using DWOSMC method for different cases.

\begin{tabular}{|c|c|c|c|}
\hline Cases & $\begin{array}{c}\text { Simulation particle } \\
\text { number }\end{array}$ & $\begin{array}{c}\text { Computational time } \\
(\mathrm{min})\end{array}$ & $\begin{array}{c}\text { Maximum relative error } \\
(\%)\end{array}$ \\
\hline \multirow{4}{*}{ I } & 500 & 0.22 & $<1$ \\
& 800 & 0.30 & $<1$ \\
\hline \multirow{4}{*}{ II } & 1000 & 0.33 & $<1$ \\
& 500 & 0.97 & 1.2 \\
& 800 & 1.86 & $<1$ \\
III & 1000 & 2.57 & $<1$ \\
& 500 & 1.41 & $<1$ \\
& 800 & 4.00 & $<1$ \\
\hline \multirow{3}{*}{ IV } & 1000 & 6.32 & $<1$ \\
& 500 & 1.79 & $<1$ \\
& 1000 & 4.42 & $<1$ \\
\hline
\end{tabular}

Table 3 shows that the more complex case is, the more computational time is needed, and for the same case, the more simulation particles used, the more computational time is needed. It is because the computational time is approximately proportional to the number of simulation particles [16]. Generally speaking, the maximum relative error is within $1 \%$ for most of the cases, which proves very high accuracy of this new DWOSMC method.

\section{Conclusions}

Results obtained from this new differentially weighted operator splitting Monte Carlo (DWOSMC) method are fully validated through four cases, including two cases for different simultaneous coagulation and condensation processes in different regimes, one case for simultaneous nucleation and coagulation processes, and one case for simultaneous nucleation, coagulation and condensation processes. The results show excellent agreement with the analytical solutions. In addition, only 500 simulation particles are good enough for the cases presented in the present study, with the maximum relative error within $1 \%$. This new DWOSMC 
method proves to have very high computational efficiency and accuracy and has high potential for solving complex aerosol dynamic problems.

\section{Acknowledgements}

The first author appreciates the help from Professor Haibo Zhao who generously share his source codes of differentially weighted Monte Carlo method [10] to support the further development of this new differentially weighted operator splitting Monte Carlo (DWOSMC) method for the present study. This work was supported by the Central Research Grants and the research studentship of The Hong Kong Polytechnic University, Hong Kong.

\section{References}

[1] Friedlander, S.K., Smoke, Dust, and Haze. Fundamentals of Aerosol Dynamics. Oxford University Press, New York, 2000.

[2] Dergaoui, H., Sartelet, K.N., Debry, E. \& Seigneur C., Modeling coagulation of externally mixed particles: Sectional approach for both size and chemical composition. Journal of Aerosol Science, 58, pp. 17-32, 2013.

[3] Liu, Y.H., He, Z. \& Chan, T.L., Three-dimensional simulation of exhaust particle dispersion and concentration fields in the near-wake region of the studied ground vehicle. Aerosol Science and Technology, 45(8), pp. 1019-1030, 2011.

[4] Chan, T.L., Liu, Y.H. \& Chan, C.K., Direct quadrature method of moments for the exhaust particle formation and evolution in the wake of the studied ground vehicle. Journal of Aerosol Science, 41(6), pp. 553-568, 2010.

[5] Fede, P., Simonin, O.\& Villedieu, P., Monte-Carlo simulation of colliding particles or coalescing droplets transported by a turbulent flow in the framework of a joint fluid-particle pdf approach. International Journal of Multiphase Flow, 74, pp. 165-183, 2015.

[6] Zhao, H. B., Zheng, C.G. \& Xu, M.H., Multi-Monte Carlo method for particle coagulation: description and validation. Applied Mathematics and Computation, 167(2), pp. 1383-1399, 2005.

[7] Zhou, K., He, Z., Xiao, M., \& Zhang, Z.Q., Parallel Monte Carlo simulation of aerosol dynamics. Advances in Mechanical Engineering, 6, pp. 1-11, 2014.

[8] Liu, S.Y. \& Chan, T.L., A stochastically weighted operator splitting Monte Carlo (SWOSMC) method for the numerical simulation of complex aerosol dynamic processes. International Journal of Numerical Methods for Heat and Fluid Flow. Accepted for publication.

[9] Debry, E., Sportisse, B. \& Jourdain, B., A stochastic approach for the numerical simulation of the general dynamics equation for aerosols. Journal of Computational Physics. 184(2), pp. 649-669, 2003. 
[10] Zhao, H.B., Kruis, F.E. \& Zheng, C.G., A differentially weighted Monte Carlo method for two-component coagulation. Journal of Computational Physics, 229(19), pp. 6931-6945, 2010.

[11] Zhao, H.B., Kruis, F.E. \& Zheng, C.G., Reducing statistical noise and extending the size spectrum by applying weighted simulation particles in Monte Carlo simulation of coagulation. Aerosol Science and Technology, 43(8), pp. 781-793, 2009.

[12] McLachlan, R.I. \& Quispel, G.R.W., Splitting methods. Acta Numerica, 11, pp. 341-434, 2002.

[13] Ramabhadran, T.E., Peterson, T.W., \& Seinfeld, J.H., Dynamics of aerosol coagulation and condensation. American Institute of Chemical Engineers Journal, 22 (5), pp. 840-851, 1976.

[14] Palaniswaamy, G. \& Loyalka S.K., Direct simulation, Monte Carlo, aerosol dynamics: coagulation and condensation. Annals of Nuclear Energy, 35(3), pp. 485-494, 2008.

[15] Maisels, A., Kruis, F.E., \& Fissan, H., Direct simulation Monte Carlo for simultaneous nucleation, coagulation, and surface growth in dispersed systems. Chemical Engineering Science, 59(11), pp. 2231-2239, 2004.

[16] Zhang, H., Liu, Q.F., Qin, B.K. \& Bo, H.L., Simulating particle collision process based on Monte Carlo method. Journal of Nuclear Science and Technology, 52(11), pp. 1393-1401, 2015. 